\title{
Molecular beam epitaxy of InN dots on nitrided sapphire
}

\author{
Yaroslav E. Romanyuk, Radu-Gabriel Dengel ${ }^{\text {a) }}$, Larissa V. Stebounova, and \\ Stephen R. Leone ${ }^{\text {b) }}$
}

Departments of Chemistry and Physics, University of California, and Lawrence Berkeley National Laboratory, Berkeley, California 94720

a) Present address: Department of Physics, University of Würzburg, Am Hubland, 97074 Würzburg, Germany

${ }^{b)}$ Corresponding author. Fax +1-510-6431376; e-mail: srl@berkeley.edu 


\begin{abstract}
A series of self-assembled InN dots are grown by radio frequency (RF) plasma-assisted molecular beam epitaxy (MBE) directly on nitrided sapphire. Initial nitridation of the sapphire substrates at $900^{\circ} \mathrm{C}$ results in the formation of a rough AlN surface layer, which acts as a very thin buffer layer and facilitates the nucleation of the $\mathrm{InN}$ dots according to the Stranski-Krastanow growth mode, with a wetting layer of $\sim 0.9 \mathrm{~nm}$. Atomic force microscopy (AFM) reveals that well-confined $\mathrm{InN}$ nanoislands with the greatest height/width at half-height ratio of 0.64 can be grown at $460^{\circ} \mathrm{C}$. Lower substrate temperatures result in a reduced aspect ratio due to a lower diffusion rate of the In adatoms, whereas the thermal decomposition of $\operatorname{InN}$ truncates the growth at $\mathrm{T}>500^{\circ} \mathrm{C}$. The densities of separated dots vary between $1.0 \times 10^{10} \mathrm{~cm}^{-2}$ and $2.5 \times 10^{10} \mathrm{~cm}^{-2}$ depending on the growth time. Optical response of the InN dots under laser excitation is studied with apertureless near-field scanning optical microscopy and photoluminescence spectroscopy, although no photoluminescence is observed from these samples. In view of the desirable implementation of InN nanostructures into photonic devices, the results indicate that nitrided sapphire is a suitable substrate for growing self-assembled InN nanodots.
\end{abstract}

Keywords: A3. Molecular beam epitaxy;

B1. Nitrides.

PACS: $\quad$ 81.15.Hi (Molecular beam epitaxy);

81.07.Ta (Quantum dots);

81.05.Ea (III-V semiconductors). 


\section{Introduction}

III-V nitrides are an important and exciting family of direct band-gap semiconductors, which has significant potential in the optoelectronics industry. A well-known example is the InGaN alloy, where self-assembled InN-rich nanoclusters formed in the nitride matrix act as electron localization centers to greatly enhance electron-hole recombination and emission efficiency of the InGaN light-emitting structures. In this respect, it is surprising that the fabrication and investigation of separate $\mathrm{InN}$ nanostructures began only recently. The main reason is the difficulty of growing pure $\mathrm{InN}$ due to its thermal decomposition, which becomes excessive above $500^{\circ} \mathrm{C}$, and its impurity incorporation problem, especially by oxygen [1].

Plasma-assisted molecular beam epitaxy (MBE) is a suitable method for producing $\mathrm{InN}$, because low growth temperatures are possible in combination with an ultra high vacuum (UHV) growth environment, which reduces impurity incorporation. Since single-crystalline InN thin films with low defect concentrations and high carrier mobilities could be grown by $\mathrm{MBE}$ at low temperatures of $450-550^{\circ} \mathrm{C}$ [1-3], it has been

proven that the energy gap of high-quality $\mathrm{InN}$ is around $0.7 \mathrm{eV}[4]$ and not the previously accepted value of 1.9-2.0 eV [5].

Besides thin film growth, the fabrication of $\mathrm{InN}$ nanodots has been studied during the last 5 years. Using MBE or metal-organic chemical vapor deposition (MOCVD), selforganized InN nanoislands are grown on different substrates, including GaN [6-12] or AlN $[13,14]$ templates on sapphire or silicon, as well as directly on Si $(111)[13,15]$. Because of the significant lattice mismatch between $\mathrm{InN}$ and the substrates, which is $8 \%$, 
$10 \%$, and $12 \%$ for $\mathrm{Si}(111), \mathrm{GaN}$, and $\mathrm{AlN}$, respectively, the growth proceeds via the Stranski-Krastanow mechanism, where most of the lattice mismatch strain is accommodated by forming dislocations in the initial wetting layer. Therefore, $3 \mathrm{D} \operatorname{InN}$ islands emerge after strain relief, and they are dislocated with a residual strain smaller than $0.6 \%$ of the lattice mismatch [16]. The island morphology depends on many parameters, such as the type of nitrogen source, In/N ratio, and substrate temperature. Typically, InN dots grown with RF plasma-assisted MBE are smaller than the ones obtained using ammonia sources [7]. Growth under nitrogen-rich conditions, i.e. In/ $\mathrm{N}<1$, results in small pyramidal islands, whereas $\mathrm{In} / \mathrm{N}>1$ provides large pillar shaped islands [8], which suggests a possible role of indium as a surfactant [12]. To explain the effects of the III/V ratio and substrate temperature, a physical model of $\mathrm{InN}$ growth was proposed [11]. The model considers the mobility of indium adatoms and the effective $\mathrm{In} / \mathrm{N}$ ratio on the surface as the main factors regulating the morphology of the $\operatorname{InN}$ dots.

For InN nanoisland growth, an AlN buffer layer also seems to be a suitable choice of substrate for several reasons. First, AlN possesses the same wurtzite structure as $\mathrm{InN}$, which allows fabrication of coherent $\mathrm{InN}$ nanostructures and avoids the formation of amorphous phases at the interface. Second, using an AlN buffer layer can significantly improve the electrical properties of $\mathrm{InN}$, in particular by decreasing the electron concentration [2,17]. Finally, an AlN layer can be formed by the direct nitridation of sapphire, a transparent, robust and well established substrate material in optoelectronics.

In this work, we test the possibility of growing InN nanodots by nitrogen plasmaassisted MBE directly on nitrided sapphire. The high-temperature nitridation results in the formation of a AlN surface layer, which will act as a very thin buffer and facilitate the 
nucleation of InN dots. Effects of the growth time and substrate temperature are implemented into the physical model to understand the results. The fabricated InN nanodots are studied by photoluminescence (PL) spectroscopy and apertureless near-field scanning optical microscopy (ANSOM), although PL is not yet observed on our samples.

\section{Experimental procedure}

InN dots were grown in a home-built MBE chamber with a base pressure of $5 \times 10^{-8} \mathrm{~Pa}$. Active nitrogen flux is generated with a SVTA RF plasma source and In is evaporated from a conventional Knudsen cell. The chamber is equipped with a low-energy electron diffraction (LEED) and Auger spectroscopy set-ups to examine surface crystal structure and chemical composition, respectively.

After standard de-greasing with organic solvents and rinsing with de-ionized water, a $c$-cut sapphire substrate is degassed at $900^{\circ} \mathrm{C}$ in vacuum for 2 hours. The substrate temperature is calibrated on a Mo-coated sapphire substrate with an optical pyrometer assuming an emissivity factor of 0.8 . The nitridation of sapphire is started by switching on the nitrogen plasma source and continues for 1 hour at $900^{\circ} \mathrm{C}$ until the LEED pattern of the substrate surface exhibits only spots of an AIN surface layer. During the nitridation, the RF plasma power is $350 \mathrm{~W}$ and nitrogen pressure is $4.5 \times 10^{-3} \mathrm{~Pa}$, corresponding to a nitrogen flow rate of about $0.3 \mathrm{sccm}$.

Following the nitridation, a series of $\mathrm{InN}$ deposits are grown at substrate temperatures from 420 to $500^{\circ} \mathrm{C}$, keeping the nitrogen pressure at $2.7 \times 10^{-3} \mathrm{~Pa}$ and $\mathrm{RF}$ power at $350 \mathrm{~W}$. The growth time is varied from 40 seconds to 4 minutes. We use a 
quartz crystal microbalance to calibrate the In flux, which is kept constant during all growth experiments, corresponding to an $\mathrm{InN}$ nominal deposition rate of $0.9 \mathrm{~nm} / \mathrm{min}$. After growth, the samples are cooled down quickly to room temperature without exposing to the nitrogen plasma. Auger spectra of as-grown samples exhibit peaks of Al, $\mathrm{N}$, In, and a small peak of $\mathrm{O}$, presumably originating from the sapphire substrate. Sonication of the samples in dilute $\mathrm{HCl}$ does not reveal any surface dissolution or dot morphology changes, which agrees with the fact that III-V nitrides are quite resistant against $\mathrm{HCl}$-based solutions [18].

Topography of the as-grown samples is analyzed ex situ with an atomic force microscope (AFM) (Multimode, Digital Instruments). The AFM images are collected in tapping mode with a pure Si-tip, radius of curvature $<10 \mathrm{~nm}$. Statistical distributions of the dot heights, widths, and volumes, as well as dot densities are investigated using a specially developed Matlab code.

Apertureless near-field scanning optical microscope (ANSOM) based on a commercial AFM (Bioscope, Veeco Instruments) is used to collect maps of the evanescent light fields excited at the sample's surface. A silicon tip oscillating perpendicularly to the sample surface scatters and re-radiates evanescent fields excited by a He-Ne laser beam $(633 \mathrm{~nm})$ in side-illumination configuration. The re-radiated light is detected by a nearby photodetector and amplified by a lock-in amplifier at the third harmonic of the tip oscillation frequency [19].

\section{Results and discussion}




\subsection{Sapphire nitridation}

Figure 1a shows an AFM image of the initial sapphire substrate surface. Monoatomic steps are clearly visible and the rms value of the surface roughness is $0.15 \mathrm{~nm}$. In order to ensure complete coverage of the sapphire surface with a uniform AlN layer, we use a high nitridation temperature of $900^{\circ} \mathrm{C}$. Exposure of the $\mathrm{Al}_{2} \mathrm{O}_{3}$ surface to nitrogen radicals created by the RF plasma source leads to the replacement of oxygen atoms and formation of the surface AlN layer shown in Fig. 1b. Small depressions appear on the surface due to the relatively high nitridation rate, which gives rise to an increased rms roughness of 0.32 nm. In-situ LEED patterns (insets in Fig.1) show a decrease of the hexagonal lattice constant in the surface plane, corresponding to the change from $\mathrm{Al}_{2} \mathrm{O}_{3}$ to $\mathrm{AlN}$. Furthermore, spots of the LEED pattern after nitridation are weaker and more diffuse than spots for the pure sapphire surface, which confirms that the nitrided surface is rougher. The presence of nano-depressions on the AlN layer slightly affects the nucleation of InN dots, as will be discussed in the next section. Nevertheless, the roughness of the as-produced AlN buffer is comparable with the rms roughness of $0.6 \mathrm{~nm}$ for a thick AlN buffer layer grown by MBE [13].

Since the penetration depth of a low energy electron beam with an energy of 100 $\mathrm{eV}$ into an inorganic solid is about 4 monolayers (ML) [20], and no residual $\mathrm{Al}_{2} \mathrm{O}_{3}$ spots are observed in the LEED patterns of the nitrided surface, we deduce that the AlN layer thickness in not less than $1.0 \mathrm{~nm}$.

\subsection{Dots nucleation}


In order to reveal the InN nucleation mechanism, we investigated how the surface morphology evolves during initial stages of deposition using AFM on samples grown for 40,60 , and $80 \mathrm{~s}$ at a substrate temperature of $460^{\circ} \mathrm{C}$. After $40 \mathrm{~s}$ of deposition (Fig. 2a), no changes in the surface morphology are visible and the rms roughness is 0.34 (what units? Above it says the AlN is rms roughness of $0.6 \mathrm{~nm}$, which his not the same?), i.e. the same as for the nitrided surface. After about $60 \mathrm{~s}$, an abrupt change is observed, and InN dots, 5-10 $\mathrm{nm}$ high, emerge (Fig. $2 \mathrm{~b}$ and $2 \mathrm{c}$ ). This behavior is typical for the StranskiKrastanow growth mode, where $\mathrm{InN}$ material, deposited during the first $60 \mathrm{~s}$, is used to form the wetting layer. Taking into account that the In flux is equivalent to the $\mathrm{InN}$ deposition rate of $0.9 \mathrm{~nm} / \mathrm{min}$, we conclude that the wetting layer thickness is about 0.9 $\mathrm{nm}$ or $\sim 3 \mathrm{ML}$. This value is in agreement with the reported wetting layers of 3.5 or $4 \mathrm{ML}$ for $\mathrm{InN}$ dots on AlN [14] or GaN [11] templates, respectively. On the other hand, a wetting layer of only $1 \mathrm{ML}$ was reported for $\mathrm{InN}$ dots on a N-polar MBE-grown GaN buffer [10]. Different values of the wetting layer thickness can be attributed to differences in the initial buffer roughness, i.e. the wetting layer is thicker for rougher buffers, because part of the deposited InN is used to "fill up" initial surface depressions.

\subsection{Effect of growth time and temperature.}

To investigate the effect of the deposition time and temperature on the morphology and density of the InN dots, a series of 9 samples were deposited at 420,460 , and $500^{\circ} \mathrm{C}$ for 2, 3, and 4 minutes. A specially developed Matlab code was employed to analyze AFM 
images of the grown samples in order to estimate the dot height, width at half-height, and density.

Figure 3 shows typical distributions of the dot height versus width at half-height for the samples grown for $3 \mathrm{~min}$ at three different substrate temperatures. Clearly there is a strong effect of the growth temperature on the dot morphology. The dot height and width at half-height are $8.6 \pm 2.7 \mathrm{~nm}$ and $39.9 \pm 6.9 \mathrm{~nm}, 13.1 \pm 4.4 \mathrm{~nm}$ and $20.4 \pm 5.1 \mathrm{~nm}$, and $10.0 \pm 2.2 \mathrm{~nm}$ and $35.0 \pm 5.4 \mathrm{~nm}$ for dots grown at $420^{\circ} \mathrm{C}, 460^{\circ} \mathrm{C}$, and $500^{\circ} \mathrm{C}$, respectively. Thus, the aspect ratio (height/width at half-height) is the smallest for the dots grown at $420^{\circ} \mathrm{C}$, reaches a maximum of 0.64 for $460^{\circ} \mathrm{C}$, and then decreases to 0.32 for $500^{\circ} \mathrm{C}$. This agrees with our AFM observations that most dots grown at $420^{\circ} \mathrm{C}$ are irregular islands with low aspect ratios. Dots grown at $460^{\circ} \mathrm{C}$ and $500^{\circ} \mathrm{C}$ have a round shape, and the highest dots are observed at $460^{\circ} \mathrm{C}$.

In order to understand the effect of the substrate temperature, we plotted the average vertical growth rate of the dots as a function of growth temperature in Fig. 4. The observed variation can be explained on the basis of a physical model for the $\mathrm{InN}$ growth on GaN proposed by Dimakis et al. [11]. Different growth regimes, distinguished by variations in indium adatom mobility and effective $\mathrm{In} / \mathrm{N}$ ratio on the surface, are identified depending on the growth temperature. At $\mathrm{T}<400^{\circ} \mathrm{C}$, the vertical growth rate approaches the nominal $\mathrm{InN}$ deposition rate of $0.9 \mathrm{~nm} / \mathrm{min}$ due to the low surface mobility of In adatoms. Starting around $400^{\circ} \mathrm{C}$, the exponentially increasing In adatom mobility gives rise to increasing vertical growth rate, which reaches its maximum at 460$470^{\circ} \mathrm{C}$. After $470^{\circ} \mathrm{C}$, the thermal decomposition of $\mathrm{InN}$ becomes significant and the aspect ratio is reduced due to the formation of a stable indium adlayer. When further 
increasing the temperature, the evaporation of $\mathrm{In}$ is expected and no $\mathrm{InN}$ deposition can be observed.

Once the dots emerge from the surface, the incident InN material is not used to increase the wetting layer thickness but rather to enlarge and multiply the $\mathrm{InN}$ dots. To illustrate this, we calculate the total volume of all $\mathrm{InN}$ nanoislands on a given surface area, convert the volume into a respective InN thickness and compare it with the expected InN thickness corresponding to the constant deposition rate of $0.9 \mathrm{~nm} / \mathrm{min}$. The dot volume is calculated considering each dot as a hemispherical cap shape. The obtained thicknesses are plotted in Fig. 5 versus the deposition time for different substrate temperatures. One can see that regardless of the deposition time, the observed surface coverage of just the dots is always $0.8-1.0 \mathrm{~nm}$ lower than the nominally deposited $\mathrm{InN}$ thickness estimated from the In flux. This difference corresponds exactly to the $0.9 \mathrm{~nm}$ thick wetting layer, which is not taken into account when calculating the dot volume. Furthermore, the surface coverage does not vary significantly with the growth temperature and increases almost linearly with the deposition time. Thus, the growth rate is limited by the In flux, typically for N-rich growth conditions.

Figure 6 shows the variation of dot density with deposition time and temperature. The dot density varies between $1.0 \times 10^{10} \mathrm{~cm}^{-2}$ and $2.5 \times 10^{10} \mathrm{~cm}^{-2}$ depending on the growth time and temperature, however, no universal trend can be observed. This behavior suggests that the dot density is predetermined by the number of existing nucleation sites on the initial substrate surface. InN dots nucleate primarily on the energetically favorable sites, like threading dislocations [16] or protrusions due to the AlN surface roughness, showing only a weak dependence on the deposition time and temperature. 


\subsection{ANSOM and photoluminescence measurements}

Apertureless near-field scanning optical microscopy is a promising method for chemical imaging of nanostructures. Near-field microscopy provides enhanced spatial resolution over the conventional diffraction limited optical microscopy. The presence of the dielectric or metal-coated tip in the illuminated spot creates a strongly confined electromagnetic field at the tip apex that can be used to excite the sample and obtain optical contrast on a sub-wavelength scale. Earlier, we demonstrated a spatial resolution of less than $30 \mathrm{~nm}$ when imaging Ga metal and InN nanoislands grown on GaN [19]. In the present study, ASNOM is used to distinguish InN dots grown on nitrided sapphire.

Figure 6 represents topography (a) and near-field (b) images of InN dots grown for 2 minutes at $500^{\circ} \mathrm{C}$. The image of the near-field is collected simultaneously with the topography of the sample as the tip is scanned across the surface. The traces below the images correspond to the dotted lines indicated in the images. A decrease in the near-field signal is observed at the place where an $\mathrm{InN}$ dot is located because the dot and surrounding surface have different dielectric constants. We observe the optical contrast in the ANSOM signal even for the dots with a height less than $2 \mathrm{~nm}$. More detailed signal interpretation is beyond the scope of this paper and will be analyzed in another publication [21].

Since high-quality InN films typically exhibit photoluminescence (PL) around 0.7 eV, we also attempted to observe a PL signal from our InN dots. Optical excitation was performed with an Ar laser at $541 \mathrm{~nm}$, and a liquid-nitrogen cooled Ge detector was used 
to monitor the possible PL. However, no significant PL signal could be detected in the range of $0.65-1.50 \mathrm{eV}$ for any of our InN samples. Instead, we observed only a wide background signal centered around $1.1 \mathrm{eV}$ that was attributed to the scattering on the sapphire substrate.

A possible reason for the strong PL quenching is the existence of a surface electron accumulation layer, which becomes especially detrimental for $\mathrm{InN}$ nanostructures [4]. To diminish the formation of the electron surface states, we deposited a $50 \mathrm{~nm}$-thick $\mathrm{TiO}_{2}$ overlay on top of the InN dots, but again, no PL signal could be detected. Our observations are similar to those described in [13], where MBE-grown InN dots were encapsulated in $\mathrm{AlN}, \mathrm{GaN}$, or $\mathrm{SiO}_{2}$. On the other hand, a recent study [22] describes a persistent PL signal around $0.8 \mathrm{eV}$ for MOCVD-grown InN dots embedded in GaN. In this respect, more detailed investigations are needed for determining the effect of interfaces and finding a suitable encapsulating material in order to explore the optical properties of InN nanodots.

\section{Conclusions}

We demonstrate that self-assembled InN dots can be grown by MBE directly on the nitrided sapphire substrates. A rough AlN surface layer formed during high-temperature sapphire nitridation promotes the nucleation of the InN dots according to the StranskiKrastanow growth mode with a wetting layer of $0.9 \mathrm{~nm}$. The substrate temperature significantly affects the dot morphology and the greatest height/width at half-height ratio of 0.64 is observed for the dots grown at $460^{\circ} \mathrm{C}$. Lower substrate temperatures result in a 
reduced aspect ratio due to lower diffusion rates of the In adatoms, whereas the thermal decomposition of $\operatorname{InN}$ truncates the growth at $\mathrm{T}>500^{\circ} \mathrm{C}$. The dot density is most likely determined by the roughness of the AlN buffer and varies between $1.0 \times 10^{10} \mathrm{~cm}^{-2}$ and $2.5 \times 10^{10} \mathrm{~cm}^{-2}$. Although ANSOM measurements reveal the difference in dielectric constants between the dots and the substrate, no PL signal characteristic of InN can be obtained, possibly due to the existence of a surface electron accumulation layer that suppresses radiative recombination. 


\section{Acknowledgements}

This study was initiated, and most of the InN samples were grown by Radu-Gabriel Dengel, member of the undergraduate student exchange program between University of Würzburg and University of California at Berkeley (curator Prof. A. Forchel). The authors thank Dr. Camelia Borca and Dr. Florent Gardillou for their help with the deposition of $\mathrm{TiO}_{2}$ overlays, and Nate Miller for the trial PL measurements. Yaroslav Romanyuk acknowledges a prospective researcher fellowship (No. PBEL2-112358) from the Swiss National Science Foundation. This work was supported by the Director, Office of Science, Office of Basic Energy Sciences, of the U.S. Department of Energy under Contract No. DE-AC02-05CH11231 


\section{References}

1. X. Wang, A. Yoshikawa, Prog. Cryst. Growth Charact. Mater. 48/49 (2004) 42.

2. H. Lu, W.J. Schaff, J. Hwang, H. Wu, G. Koley, L.F. Eastman LF, Appl. Phys. Lett. 79 (2001) 1489.

3. Y. Nanishi, Y. Saito, T. Yamaguchi, Jap. J. Appl. Phys. 42 (2003) 2549.

4. W. Walukiewicz, J.W. Ager III, K.M. Yu, Z. Liliental-Weber, J. Wu, S.X Li, R.E. Jones, J.D. Denlinger, J. Phys. D: Appl. Phys. 39 (2006) R83.

5. T.L. Tansley, C.P. Foley, J. Appl. Phys. 59 (1986) 3241.

6. Y.F. Ng, Y.G. Cao, M.H. Xie, X.L. Wang, S.Y. Tong, Appl. Phys. Lett. 81 (2002) 3960 .

7. C. Nörenberg, R. A. Oliver, M. G. Martin, L. Allers, M. R. Castell, G. A. D. Briggs, Phys. Stat. Sol. (A) 194 (2002) 536.

8. Y. G. Cao, M. H. Xie, Y. Liu, Y. F. Ng, H. S. Wu, Appl. Phys. Lett. 83 (2003) 5157.

9. O. Briot, B. Maleyre, S. Ruffenach, Appl. Phys. Lett. 83 (2003) 2919.

10. A. Yoshikawa, N. Hashimoto, N. Kikukawa, S. B. Che, Y. Ishitani, Appl. Phys. Lett. 86 (2005) 153115.

11. E. Dimakis, E. Illiopoulos, K. Tsagarki, A. Georgakilas, Appl. Phys. Lett. 86 (2005) 133104.

12. B. Liu, T. Kitajima, D. Chen, S.R. Leone, J. Vac. Sci. Technol. A 23 (2005) 304.

13. S. Ruffenach, B. Maleyre, O. Briot, B. Gil, Phys. Stat. Sol. (C) 2 (2005) 826. 
14. C.-H. Shen, H.-W. Lin, H.-M. Lee, C.-L. Wu, J.-T. Hsu, S. Gwo, Thin Solid Films 494 (2006) 79.

15. C. Nörenberg, R. A. Oliver, M. G. Martin, L. Allers, M. R. Castell, G. A. D. Briggs, J. Phys. D: Appl. Phys. 35 (2002) 615.

16. J.G. Lozano, A.M. Sánchez, R. García, D. González, D. Araújo, S. Ruffenach, O.Briot, Appl. Phys. Lett. 87 (2005) 263104.

17. B. Maleyre, S. Ruffenach, O.Briot, B. Gil, A. Van der Lee, Superlattices Microstruct. 36 (2004) 517.

18. C.B. Vartuli, S.J. Pearton, C.R. Abernathy, J.D. MacKenzie, F. Ren, J.C. Zolper, R.J. Shul, Solid-State Electron. 41 (1997) 1947.

19. Z.H. Kim, B. Liu, S.R. Leone, J. Phys. Chem. B 109 (2005) 8503.

20. M.P. Seah, W.A. Dench, Surf. Interface Anal. 1 (1979) 2.

21. L. V. Stebounova, R.-G. Dengel, Y. E. Romanyuk, S.R. Leone, in preparation.

22. W.C. Ke, C.P. Fu, C.Y. Chen, L.Lee, C.S. Ku, W.C. Chou, W.-H. Chang, M.C. Lee, W.K. Chen, W.J. Lin, Y.C. Cheng, Appl. Phys. Lett. 88 (2006) 191913. 
Figure captions

Fig. 1. AFM images of the (0001) sapphire surface before (a) and after (b) the nitridation at $900^{\circ} \mathrm{C}$ for $1 \mathrm{~h}$. Insets depict corresponding LEED patterns taken at the electron beam energy of $100 \mathrm{eV}$.

Fig. 2. AFM images showing the nucleation of $\mathrm{InN}$ dots on the nitrided sapphire. Growth time is a) $40 \mathrm{~s}$, b) $60 \mathrm{~s}$, and c) $80 \mathrm{~s}$.

Fig. 3. Aspect ratio plots for $\mathrm{InN}$ dots deposited for 3 minutes at the substrate temperature of a) $420^{\circ} \mathrm{C}$, b) $460^{\circ} \mathrm{C}$, and c) $500^{\circ} \mathrm{C}$. Each plot contains data points obtained from two $1 \times 1 \mu \mathrm{m}$ AFM images. Fig. 3 has several different symbols, but there is no explanation of what these symbols are?

Fig. 4. Vertical growth rate for $\mathrm{InN}$ dots as a function of the deposition temperature. Solid line shows an expected vertical growth rate for the temperature range of $250-550^{\circ} \mathrm{C}$. Two guiding lines schematically represent limiting factors for the vertical growth: surface mobility of In adatoms (dashed line) and thermal decomposition of InN (dash-dotted line).

Fig. 5. Nominal InN thickness as a function of the deposition time for different growth temperatures. The nominal thickness is calculated from the total volume of $\mathrm{InN}$ dots. Dashed line represents the expected InN coverage limited by the In flux. 
Fig. 6. Variation of the InN dot density with deposition temperature and time. Only dots with heights greater than $1.5 \mathrm{~nm}$ are considered.

Fig. 7. AFM (a) and ANSOM (b) images of InN dots grown for 2 minutes at $500^{\circ} \mathrm{C}$. Profiles show the change in the height and near-field signals along the dashed lines. 
Figure 1

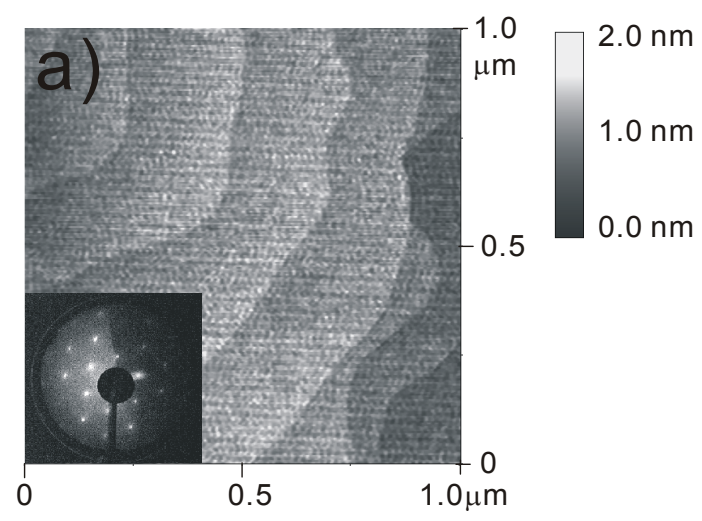

Y.E. Romanyuk et al.

J. Cryst. Growth

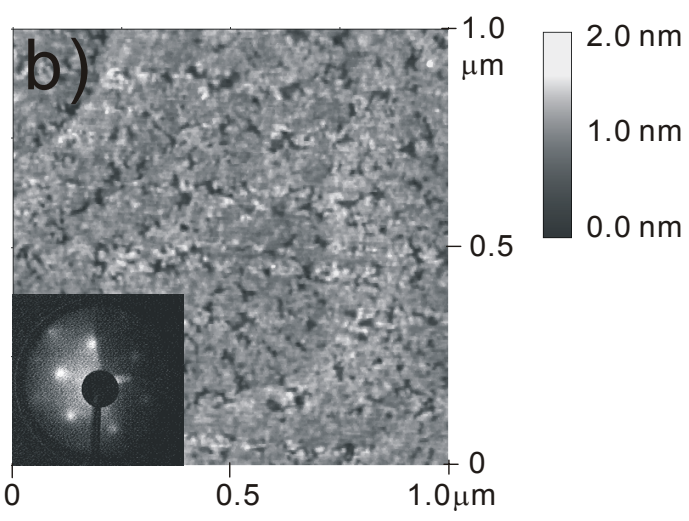


Figure 2
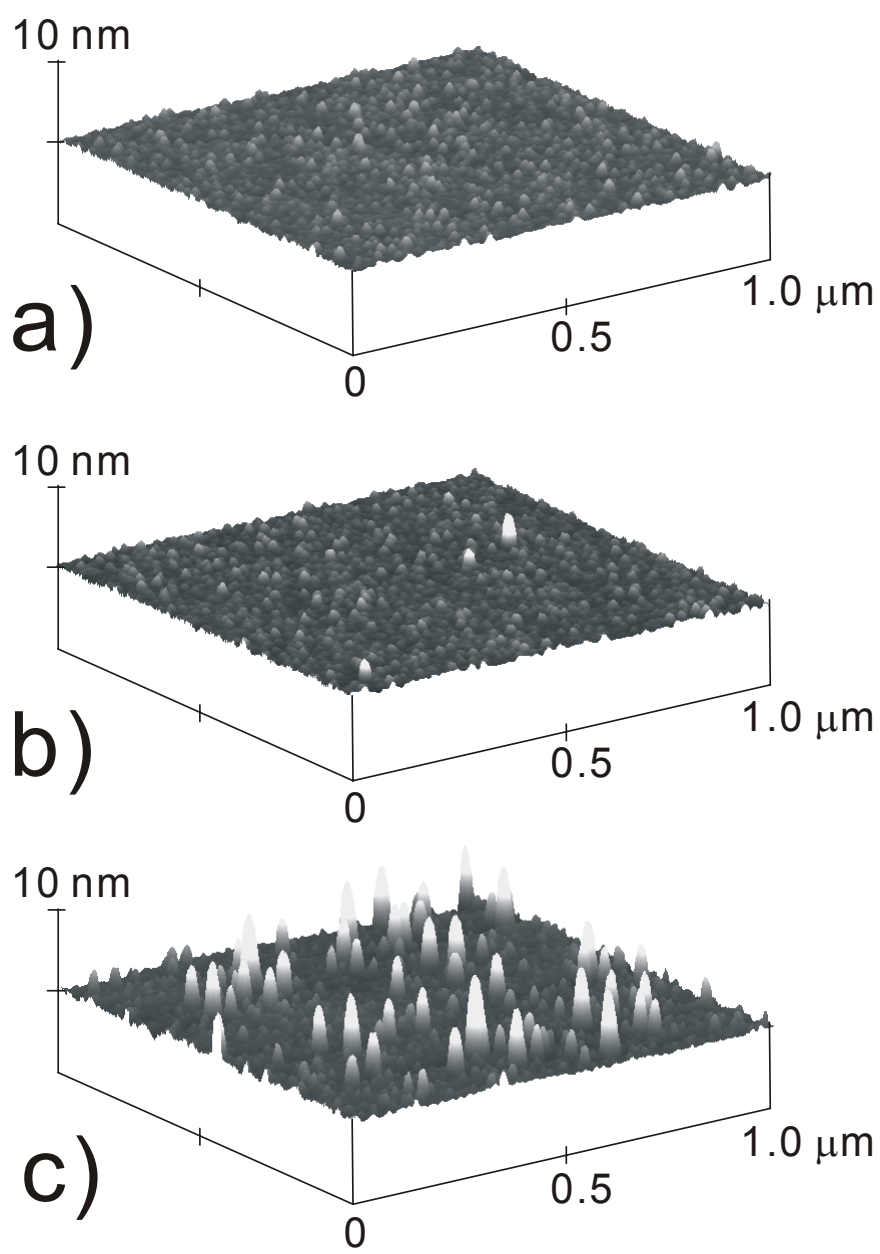


\section{Figure 3}

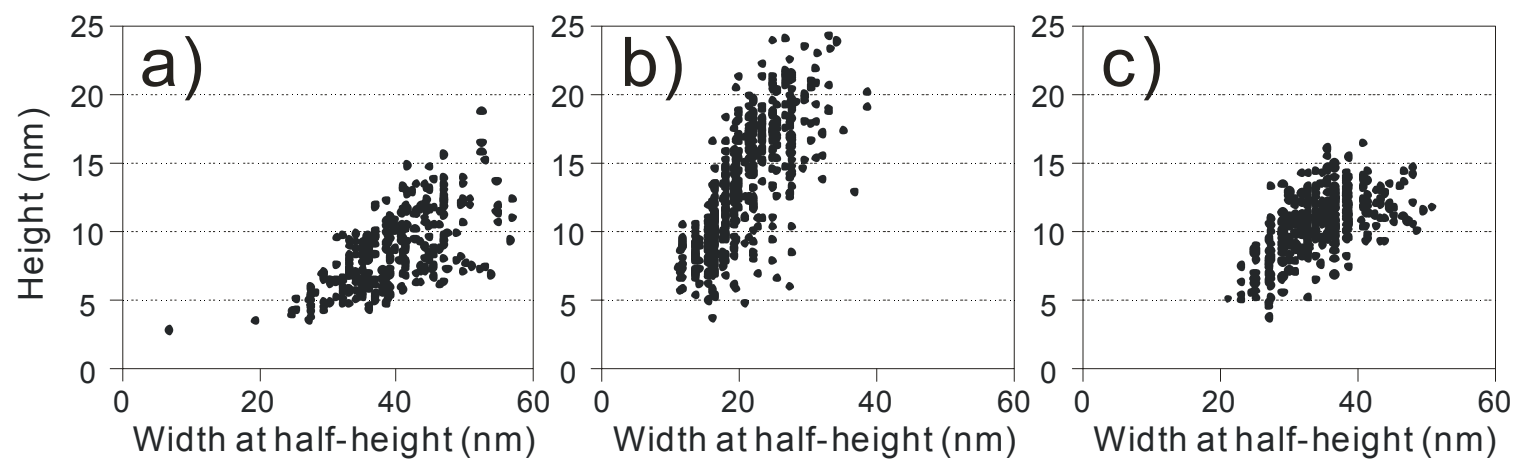


Figure 4

Y.E. Romanyuk et al.

J. Cryst. Growth

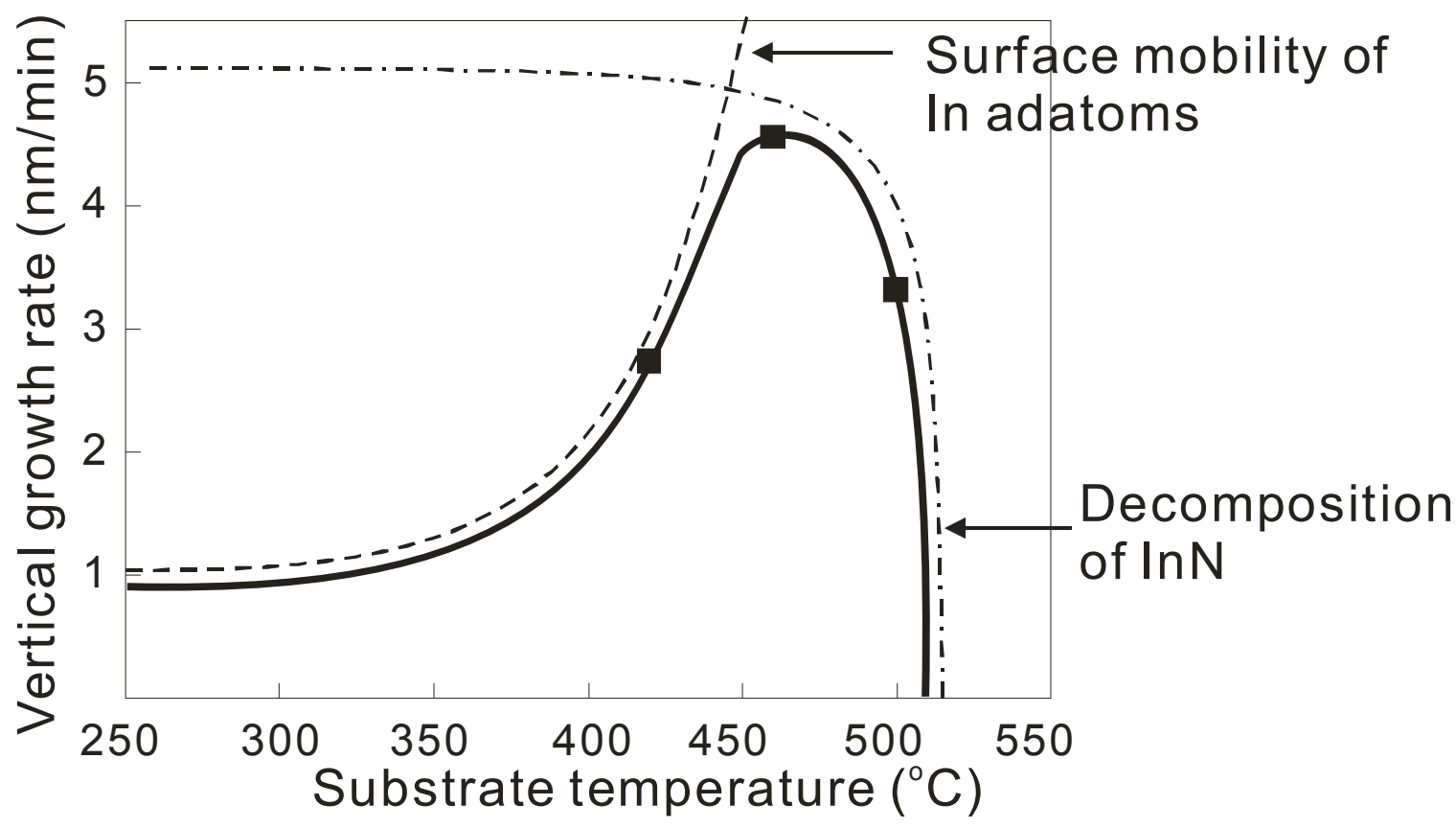


Figure 5

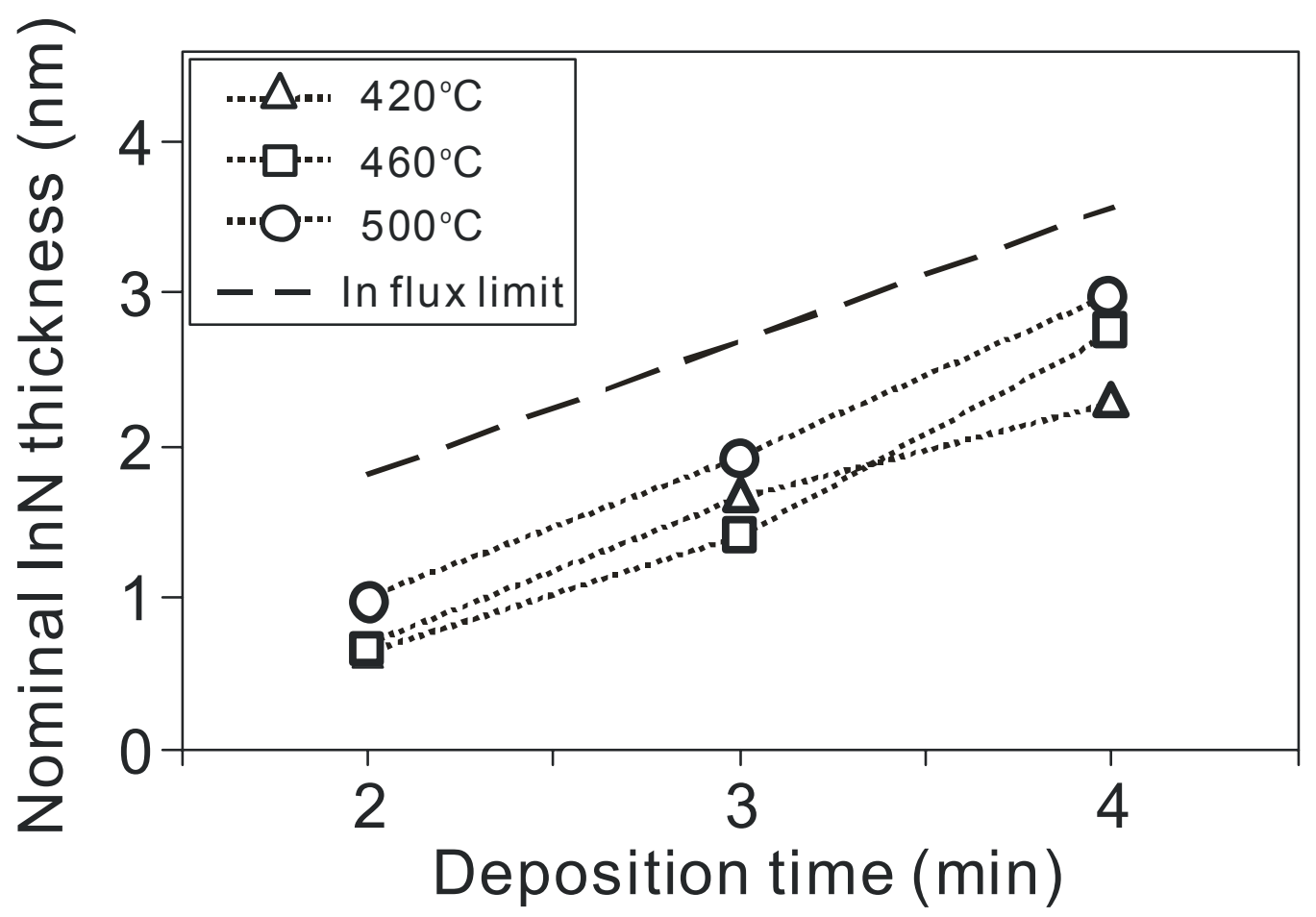




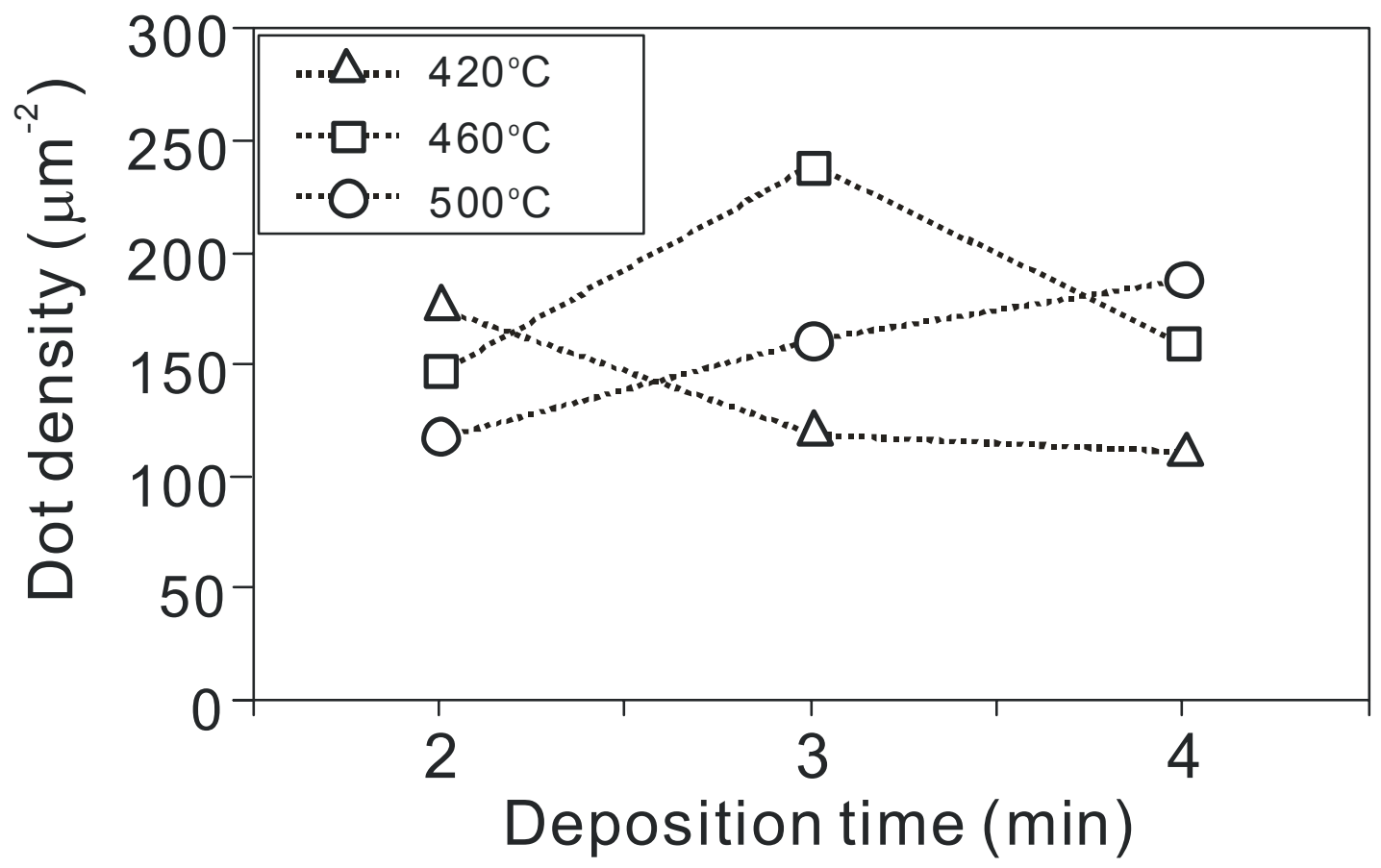



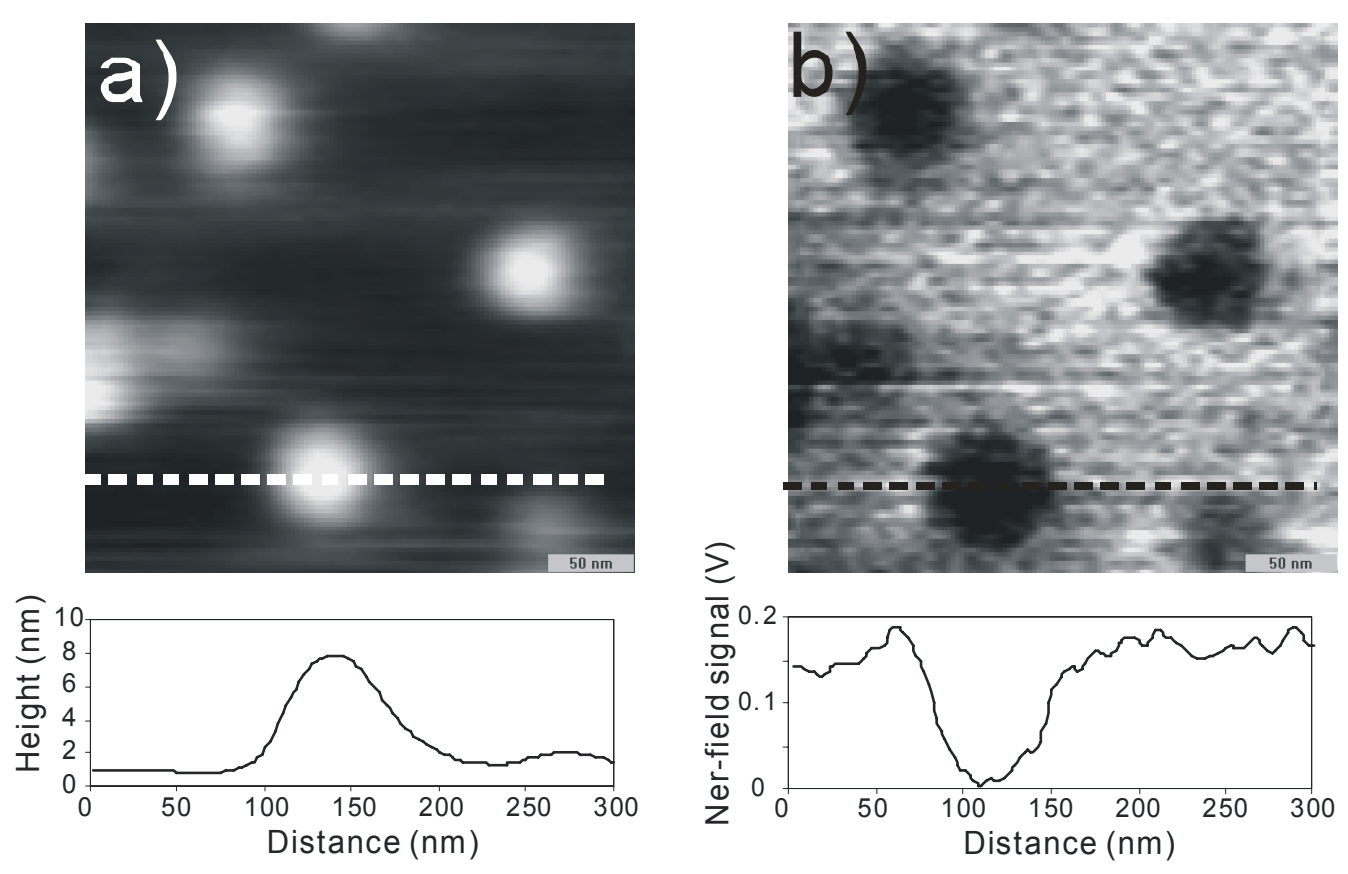